\title{
Developing a Screening Procedure During the COVID-19 Pandemic: Process and Challenges Faced by a Low-Incidence Area
}

OPEN ACCESS

Edited by:

Reza Lashgari,

Shahid Beheshti University, Iran

Reviewed by:

Bin Lin,

Zhejiang University, China

Guang Yang,

Imperial College London,

United Kingdom

*Correspondence:

Jian-Wei Wang

dr_jianweiwang@sina.com

Specialty section:

This article was submitted to

Pulmonary Medicine,

a section of the journal

Frontiers in Medicine

Received: 17 January 2021 Accepted: 20 October 2021 Published: 24 December 2021

Citation:

Tang W, Wang F, Wang J-W, Huang $Y$,

Liu L, Zhao S-J, Zhao X-M and Wu N (2021) Developing a Screening Procedure During the COVID-19

Pandemic: Process and Challenges

Faced by a Low-Incidence Area.

Front. Med. 8:654754.

doi: 10.3389/fmed.2021.654754

\begin{abstract}
Wei Tang ${ }^{1}$, Fei Wang ${ }^{2}$, Jian-Wei Wang ${ }^{1 *}$, Yao Huang ${ }^{1}$, Li Liu ${ }^{1}$, Shi-Jun Zhao ${ }^{1}$, Xin-Ming Zhao ${ }^{1}$ and Ning $W^{1,3}$
\end{abstract}

\begin{abstract}
${ }^{1}$ Department of Diagnostic Radiology, National Cancer Center, National Clinical Research Center for Cancer, Cancer Hospital, Chinese Academy of Medical Sciences and Peking Union Medical College, Beijing, China, ${ }^{2}$ Office of Cancer Screening, National Cancer Center, National Clinical Research Center for Cancer, Cancer Hospital, Chinese Academy of Medical Sciences and Peking Union Medical College, Beijing, China, ${ }^{3}$ Department of PET-CT Center, National Cancer Center, National Clinical Research Center for Cancer, Cancer Hospital, Chinese Academy of Medical Sciences and Peking Union Medcial College, Beijing, China
\end{abstract}

Purpose: To summarize the imaging results of COVID-19 pneumonia and develop a computerized tomography (CT) screening procedure for patients at our institution with malignant tumors.

Methods: Following epidemiological investigation, 1,429 patients preparing to undergo anti-tumor-treatment underwent CT scans between February 17 and April 16, 2020. When CT findings showed suspected COVID-19 pneumonia after the supervisor radiologist and the thoracic experience radiologist had double-read the initial CT images, radiologists would report the result to our hospital infection control staff. Further necessary examinations, including the RT-PCR test, in the assigned hospital was strongly recommended for patients with positive CT results. The CT examination room would perform sterilization for $30 \mathrm{~min}$ to $1 \mathrm{~h}$. If the negative results of any suspected COVID-19 pneumonia CT findings were identified, the radiologists would upload the results to our Hospital Information Systems and inform clinicians within $2 \mathrm{~h}$.

Results: Fifty $(0.35 \%, 50 / 1,429)$ suspected pneumonia cases, including 29 males and 21 females (median age: 59.5 years old; age range $27-79$ years), were identified. A total of $34.0 \%(17 / 50)$ of the patients had a history of lung cancer and $54.0(27 / 50)$ underwent chemotherapy or targeted therapy. Forty-six patients (92.0\%) had prior CT scans, and 35 patients $(76.1 \%)$ with suspected pneumonia were newly seen (median interval time: 62 days). Sub-pleura small patchy or strip-like lesions most likely due to fibrosis or hypostatic pneumonia and cluster of nodular lesions were the two main signs of suspected cases on CT images (34, 68.0\%). Twenty-seven patients (54.0\%) had, at least once, follow-up CT scan (median interval time: 18.0 days). Only one patient had an increase in size (interval time: 8 days), the immediately RT-PCR test result was negative.

Conclusion: CT may be useful as a screening tool for COVID-19 based on imaging features. But the differential diagnosis between COVID-19 and other pulmonary infection and/or non-infectious disease is very difficult due to its overlapping imaging features. The confirmed diagnosis of the COVID-19 infection should be based on the etiologic 
eventually. The cancer patients at a low-incidence area would continue treatment by screening carefully before admission.

Keywords: COVID-19, screening, computerized tomography (CT), healthcare [MeSH], hospital (clinic)-clinic corporation

\section{INTRODUCTION}

Since December 2019, the Novel Coronavirus (COVID-19) has caused an outbreak of pneumonia and has rapidly spread across the globe. On 11 March 2020, the WHO, declared COVID-19 to be a pandemic (1). Globally, as of 2:10 p.m. CET, 13 March 2021 , there have been $118,754,336$ confirmed cases of COVID19 , including 2,634,370 deaths, reported to WHO. While the number of confirmed and suspected cases continues to grow, the rate of increase is now gradually declining in some areas. On March 19, China reported no new domestic cases for the first time, and the first prevailing peak period of the pandemic was over. Thanks to the efforts of the Chinese government, numerous measures such as preventing the spread and import of the infection, strict restrictions on the gathering of large numbers of people, disinfecting public areas, and taking charge of symptomatic people (such as those with fever and/or cough) has led to Beijing, the capital of China, maintaining its status up until mid-April, 2020 as a low-incidence area of COVID-19 infections during the period of outbreak in China.

The epidemiologic, laboratory, and clinical features of COVID-19 pneumonia have been described (2-5). Because a curative vaccine has not yet been developed, early detection and efficient control of the route of transmission (i.e., isolation of suspected cases, and disinfection) remain the most effective ways to fight the outbreak. The WHO recommends suspected cases be screened for the virus with nucleic acid amplification tests (NAAT) (6), such as reverse-transcriptase polymerase chain reaction (RT-PCR). Laboratory detection is time-consuming and may not be available for all those with a suspected infection owing to the shortage of test kits for SARS-CoV-2. These challenges increase the risk of spread by free movement of people highly suspected of carrying the disease. In addition, the laboratory test can give false negative results. CT is considered the firstline imaging modality in highly suspected cases and is helpful in monitoring imaging changes during treatment. Therefore, CT has been identified as an efficient clinical diagnostic tool for people with suspected COVID-19 (7).

Sensitivity and specificity of chest CT for COVID-19 are reported to range from 80 to $90 \%$ and 60 to $70 \%$, respectively ( 8 , 9 ). The diagnosis and treatment program (6th version), published by the National Health Commission of the People's Republic of China (10), defined the diagnosis of viral pneumonia based on radiologic features by radiologists as one of the diagnostic criteria for COVID-19.

The Cancer Hospital Chinese Academy of Medical Sciences (CHCAMS) \& National Cancer Center of China (NCC) is one of the leading specialist cancer hospitals of China. NCC has over 1,000,000-patient output annually. In 2019, 79,398 X-ray, 210,503 CT, and 49,988 MRI examinations were performed in the center. Patients with cancer have an increased risk of complication and death during or after treatment related to COVID-19 infection (11, 12). During the outbreak period, a large proportion of the examinations, diagnosis, treatments and curative effect evaluations of patients were delayed. It is very important for the patients with malignant tumor that the prevention and control of COVID-19 infection, especially when they undergo therapies such as chemotherapy, radiotherapy, targeted therapy or immunotherapy. Our hospital has made any efforts to minimize any possibilities of in-hospital transmission to ensure a safe environment for both patients and staff. In order to make the patients with malignant tumor get the necessary treatment on time, the infection control committee of NCC/CHCAMS decided to use CT to screen the COVID-19 pneumonia for pre-treatment patients as a necessary examination on February 16, 2019.

To our knowledge, few articles which focuses on the COVID19 CT screening for cancer patients had been published (13). Now, we will want to share our experience and understanding with medical peers, which will contribute directly to the accumulation of experience in other medical facilities.

\section{MATERIALS AND METHODS STUDY Design and Patients}

The questionnaire survey which designed by our hospital infection control team were written by each pre-anti-tumor treatment patients. This questionnaire included personal information (gender, age, telephone number, home or permanent address, exact date of arrival Beijing, and duration from arrival in Beijing to the current date) and epidemiological investigation. The epidemiological investigation included (10): (i) whether the patient had been to Hubei Province within the past 2 weeks; (ii) whether the patient had taken public transport which passed through Hubei Province within the past 2 weeks; (iii) whether the patient had had contact with anybody from Hubei Province within the past 2 weeks; (iv) whether the patient had been abroad or had had contact with anybody who coming overseas; (v) whether the patient had had contact with COVID-19 confirmed or suspected cases; (vi) appearance or worsening of symptoms such as fever and body temperature; (vii) whether the patient had taken any antipyretics during the fever; and (viii) whether the patient displayed typical symptoms such as fever, sore throat, nasal congestion, malaise, headache, myalgia, or dyspnea. Given that our hospital was not a designated hospital for COVID-19 infection patients, if patients or those accompanying them gave any positive responses, they would be declined entry. If the patients or those accompanying them had a fever higher than $37.3^{\circ} \mathrm{C}$, and had no alternative explanation for these symptoms, COVID-19 testing via a SARS-CoV-2 quantitative 


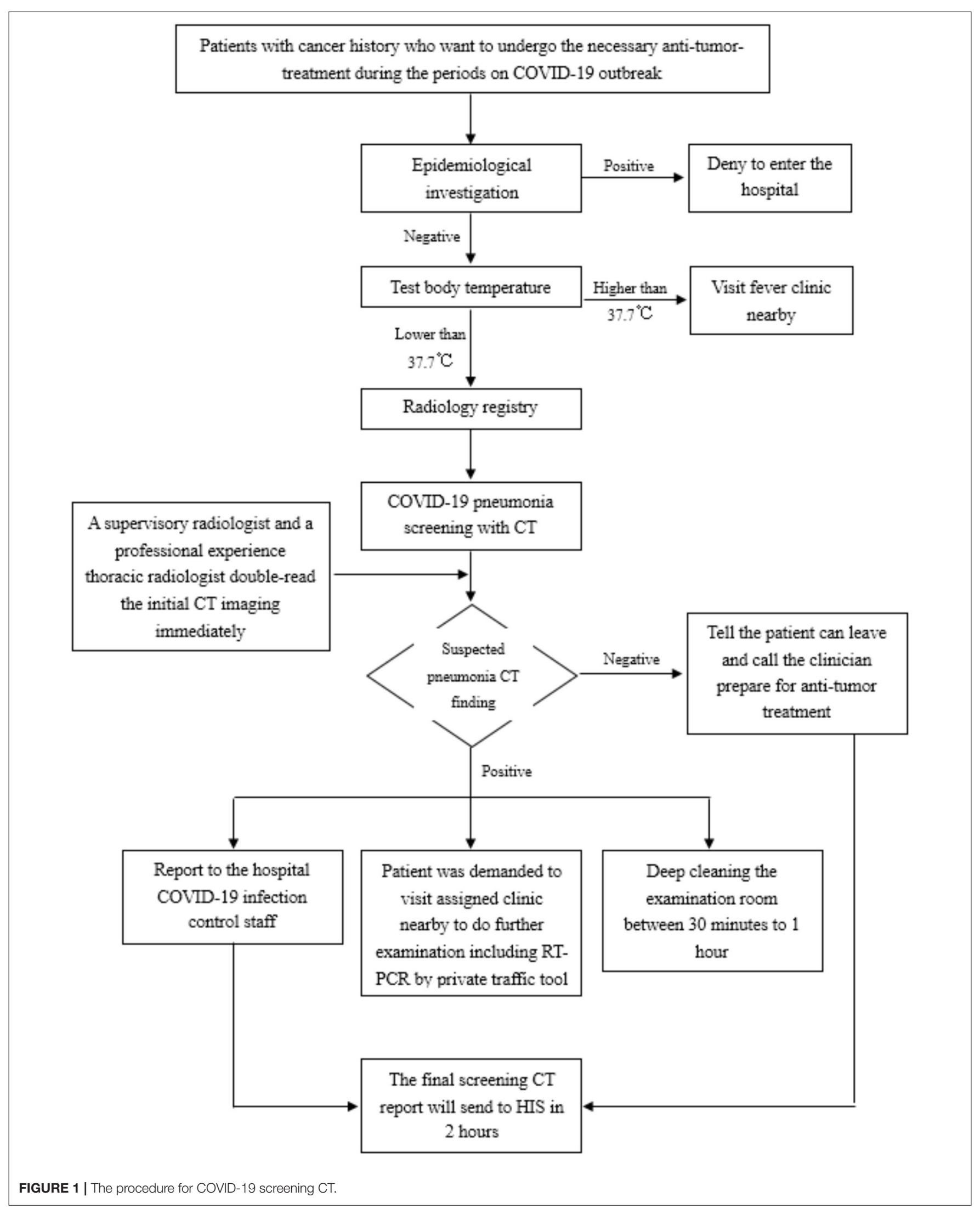


PCR nasopharyngeal swab was sent in addition to a respiratory pathogen panel PCR at the nearby fever clinic.

Finally, in this center, a total of 1,429 patients with cancer history underwent the CT procedure and routine blood test between February 17 and April 16, 2020. All patients denied any direct exposure history to Wuhan or to people who had a direct exposure history to Wuhan (i.e., long-term exposure history to Wuhan, or travel to Wuhan before diagnosis) and no one showed any symptoms associated with COVID-19.

\section{Imaging Technique}

All patients underwent scanning with the following four scanners: Optima 660, LightSpeed VCT, Discovery 750 HD (GE Healthcare, Milwaukie, USA), and Somatom Edge (Siemens Healthcare, Erlangen, Germany). Instead, the routine CT scan of Low- dose CT scan was used. The acquisition parameters were set at $120 \mathrm{kVp}$; 150-250 mAs; pitch, 0.938-1.0; and collimation, $0.625 \mathrm{~mm}$. All imaging data were reconstructed by use of a standard reconstruction algorithm with a slice thickness of $0.625-1.0 \mathrm{~mm}$. All patients were scanned in the supine position wild holding their breath at the end of inspiration. The field of view was set from the apex to the base of the lungs. Images were sent to the Picture Archiving and Communication System (PACS) system to be read by the radiologists.

\section{Computed Tomography Scan Procedure and Imaging Interpretation}

To detect suspected COVID-19 on CT imaging, we arranged for several Supervisory Radiologists (SR) to read the CT images immediately after completion of the scan. The SR confirmed whether the CT images showed signs of any type of pneumonia, including bacterial pneumonia, viral pneumonia, and other types, including suspected COVID-19. Comparison was made with prior CT images, in order to confirm whether the lesion was newly seen, persistently existed, or had changed in size or density. All images were viewed with both lung (width, 1,500 HU; level, $-700 \mathrm{HU}$ ) and mediastinal (width, $350 \mathrm{HU}$; level, $40 \mathrm{HU}$ ) settings. The patient was not permitted to leave the scan room until the technologist of radiology (TR) had obtained permission from the SR. If any CT images showed suspected pneumonia, the SR would ask one of the Professor Radiologists (PR, Y. Huang, JW. Wang, SJ. Zhao, and L. Liu, each of whom have over 10 years of thoracic-imaging diagnosis experience) to immediately read the images again. If the PR confirmed the suspected diagnostic of viral pneumonia, the SR would immediately report the details, including the CT findings and blood test results, to the clinician and the hospital infection control staff. The clinician would then order immediate isolation of the patient for clinical monitoring and treatment. The TR would request the patients attend the nearby fever clinic (given that our center does not have a fever clinic) while avoiding use of public transportation to do the further examination including RT-PCR. The hospital infection control staff would document the information to follow up the results of the patient. Sterilization would be performed: room downtime was typically $30 \mathrm{~min}$ to $1 \mathrm{~h}$ for room decontamination and passive air exchange. In addition, the final CT report would be double-read by a further two radiologists. The
TABLE 1 | Malignant tumor and the treatment history of the patients.

\begin{tabular}{|c|c|c|c|c|}
\hline & $\begin{array}{l}\text { Surgical } \\
\text { resection } \\
(n=26)\end{array}$ & $\begin{array}{l}\text { Chemotherypy/ } \\
\text { Targettherypy } \\
(n=27)\end{array}$ & $\begin{array}{l}\text { Radiotherapy } \\
\qquad(n=8)\end{array}$ & $\begin{array}{c}\text { Interventinoal } \\
\text { therapy } \\
(n=1)\end{array}$ \\
\hline $\begin{array}{l}\text { Lung cancer } \\
(n=17)\end{array}$ & 6 & 12 & 1 & 0 \\
\hline $\begin{array}{l}\text { Breast cancer } \\
(n=9)\end{array}$ & 8 & 1 & 1 & 0 \\
\hline $\begin{array}{l}\text { Esophagus cancer } \\
(n=4)\end{array}$ & 2 & 2 & 2 & 0 \\
\hline $\begin{array}{l}\text { Gastric cancer } \\
(n=3)\end{array}$ & 2 & 1 & 0 & 0 \\
\hline $\begin{array}{l}\text { Ovarian cancer } \\
(n=3)\end{array}$ & 2 & 3 & 0 & 0 \\
\hline $\begin{array}{l}\text { Endometrial } \\
\text { cancer }(n=2)\end{array}$ & 1 & 1 & 1 & 0 \\
\hline $\begin{array}{l}\text { Hepatocellular } \\
\text { carcinoma }(n=2)\end{array}$ & 1 & 0 & 0 & 1 \\
\hline $\begin{array}{l}\text { Non-Hodgkin } \\
\text { Lymphoma } \\
(n=2)\end{array}$ & 0 & 2 & 0 & 0 \\
\hline $\begin{array}{l}\text { Gallbladder } \\
\text { carcinoma }(n=1)\end{array}$ & 1 & 1 & 0 & 0 \\
\hline $\begin{array}{l}\text { Colon cancer } \\
(n=1)\end{array}$ & 1 & 0 & 0 & 0 \\
\hline $\begin{array}{l}\text { Hodgkin } \\
\text { lymphoma }(n=1)\end{array}$ & 0 & 1 & 0 & 0 \\
\hline $\begin{array}{l}\text { Cervical cancer } \\
(n=1)\end{array}$ & 0 & 1 & 1 & 0 \\
\hline $\begin{array}{l}\text { Thymic carcinoma } \\
(n=1)\end{array}$ & 0 & 1 & 1 & 0 \\
\hline $\begin{array}{l}\text { Clear cell } \\
\text { carcinoma of } \\
\text { kidney }(n=1)\end{array}$ & 1 & 0 & 0 & 0 \\
\hline $\begin{array}{l}\text { Nasopharyngeal } \\
\text { carcinoma }(n=1)\end{array}$ & 0 & 1 & 1 & 0 \\
\hline $\begin{array}{l}\text { Olfactory } \\
\text { neuroblastoma } \\
(n=1)\end{array}$ & 1 & 0 & 0 & 0 \\
\hline
\end{tabular}

imaging features defined in a previous study (14): ground-glass opacities (GGO), consolidation, mixed GGO and consolidation, centrilobular nodules, architectural distortion, cavitation, treein-bud, bronchial wall thickening, reticulation, subpleural bands, traction bronchiectasis, intrathoracic lymph node enlargement, vascular enlargement in the lesion, and pleural effusions (Figure 1 refers to the procedure for COVID-19 screening CT).

\section{Follow-Up CT Scan}

We defined three types of imaging changes: no change, progressive change, and improvement change. No change referred to no obvious changes presented in the chest CT progressive change referred to the presence of new lesions or the presence of extent involvement area during treatment; and improvement change referred to the continually absorbed abnormities. We also evaluated the duration of imaging progress, which was calculated from the time of baseline CT or the time 


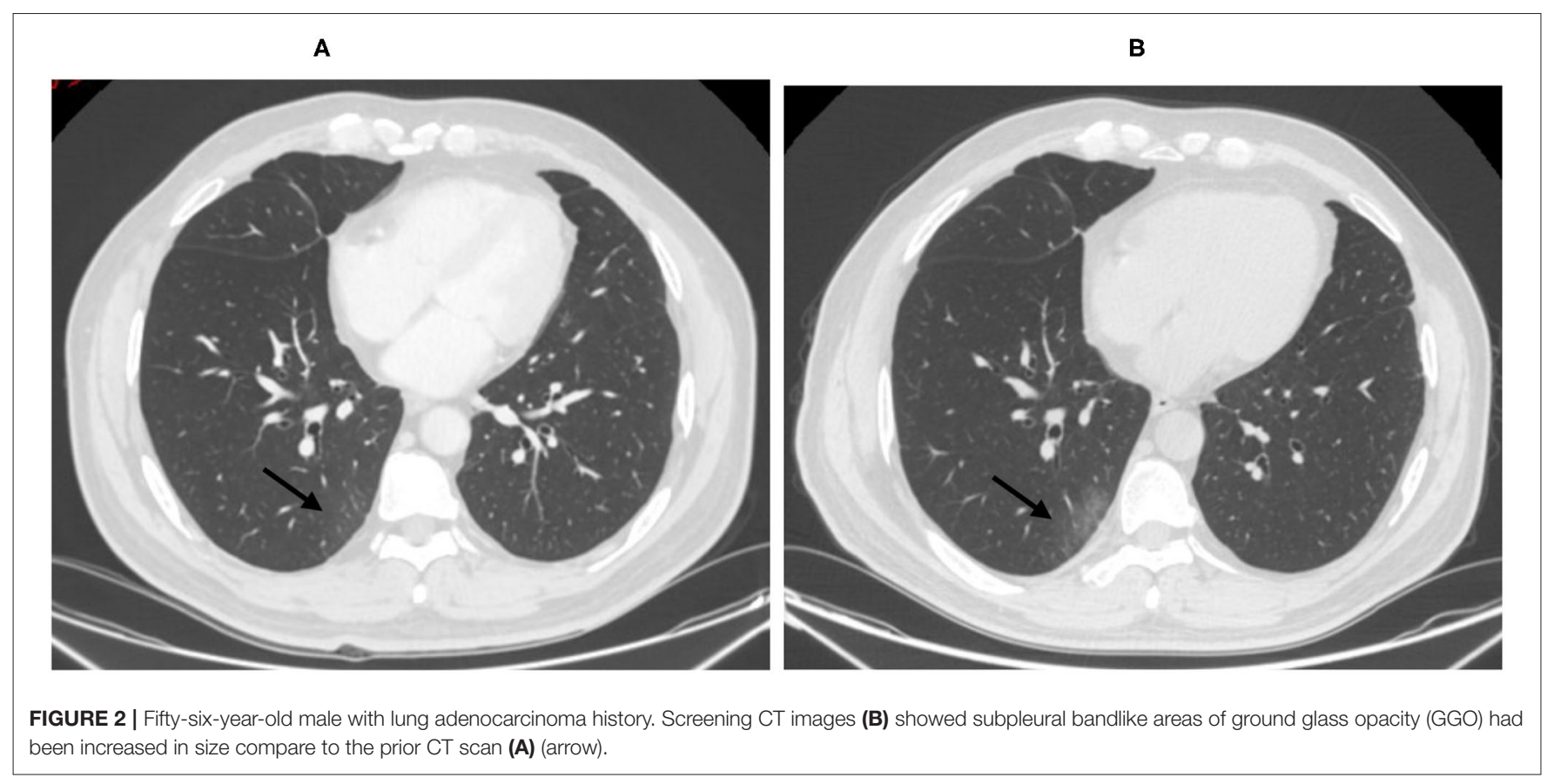

of CT showing new lesions to that of the CT showing abnormal imaging findings.

\section{RESULTS}

\section{Patients}

Among the 1,429 COVID-19 infection screening cases for pre-inpatients, 50 cases $(0.35 \%, 50 / 1,429)$ of suspected virus pneumonia in CT imaging, which had been double-read by SR and PR, were reported to the hospital infection control staff. The total of 50 suspected virus pneumonia patients (29 males, 21 females; median 59.5 years old; age range 2779 years) were included in analysis and the cancer history. Treatment history before CT scan were listed in Table 1. A total of $34.0 \%(17 / 50)$ of the patients had a history of lung cancer, $18.0 \%(9 / 50)$ had a history of breast cancer. Fifty-two percentage (26/50) patients underwent surgical resection, 54.0\% (27/50) underwent chemotherapy or targeted therapy, $16.0 \%$ (8/50) underwent radiotherapy, and one patient $(4.0 \%)$ with hepatocellular carcinoma underwent interventional therapy. Thirty-six patients (72.0\%) were natives of Beijing and 14 patients (28.0\%) were from outside of Beijing, including one patient from Hubei Province. They had complied with 2-week home quarantine requirements before the $\mathrm{CT}$ scan. All available clinical, laboratory examinations, and epidemic characteristics were collected.

\section{Initial COVID-19 Screening CT Scan}

In our retrospective cohort of 50 patients, 46 patients (92.0\%) had prior CT scans. Thirty-five patients $(76.1 \%, 35 / 46)$ with suspected pneumonia were newly seen (interval time: 26-145 days, median: 62 days), eight patients $(17.4 \%, 8 / 46)$ had slightly increased in size or density (interval time: 13-144 days, median:
74 days), two patients $(4.3 \%, 2 / 46)$ had no change, and one $(2.2 \%, 1 / 46)$ had increased in size when compared with the recent examination.

In COVID-19 screening CT, all lung segments may be involved; there was a slight predilection for the left lower lobe (30 (33.0\%) of 91 affected segments among all patients). Involvement of lower lobes (right 48.0 vs. left 60.0\%) was more than that of the upper lobes (right 20.0 vs. left $40.0 \%$ ). Twenty-nine (58.0\%) patients had single lobe involvement, 10 (20.0\%) patients had involvement of two lobes, and 11 (22.0\%) patients had three or more lobes involved. The lesions were predominantly peripheral and subpleural in 29 (58.0\%) patients. Sub-pleura small patchy (Figure 2) or strip-like lesions most likely due to fibrosis or hypostatic pneumonia and cluster of nodular lesions (Figure 3) were two main signs of suspected cases on CT images $(34,68.0 \%)$. Fourteen cases $(28.0 \%, 14 / 50)$ manifested GGO (Figure 4) and consolidation (Figure 5). One case $(2.0 \%, 1 / 50)$ manifested interstitial inflammation (Figure 6) and another case $(2.0 \%, 1 / 50)$ manifested diffuse small airway lesions most likely due to respiratory bronchiolitis. No patients had mediastinal lymphadenopathy or pleural effusion. Cavitation and tree-in-bud were absent in our cohort. In addition, a part-solid pulmonary nodule with a primary lung cancer imaging feature was identified in a patient with a history of esophagus cancer.

\section{Follow Up COVID-Screening CT Scan}

A total of 85 chest CT examinations on 27 patients had been performed with at least once follow up CT scan as of April 16, 2020. The mean number of CT examinations per patient was $1.7(85 / 50)$. The mean time between the initial and follow up CT studies was 18.0 days (range, 6-53 days). Only one patient 


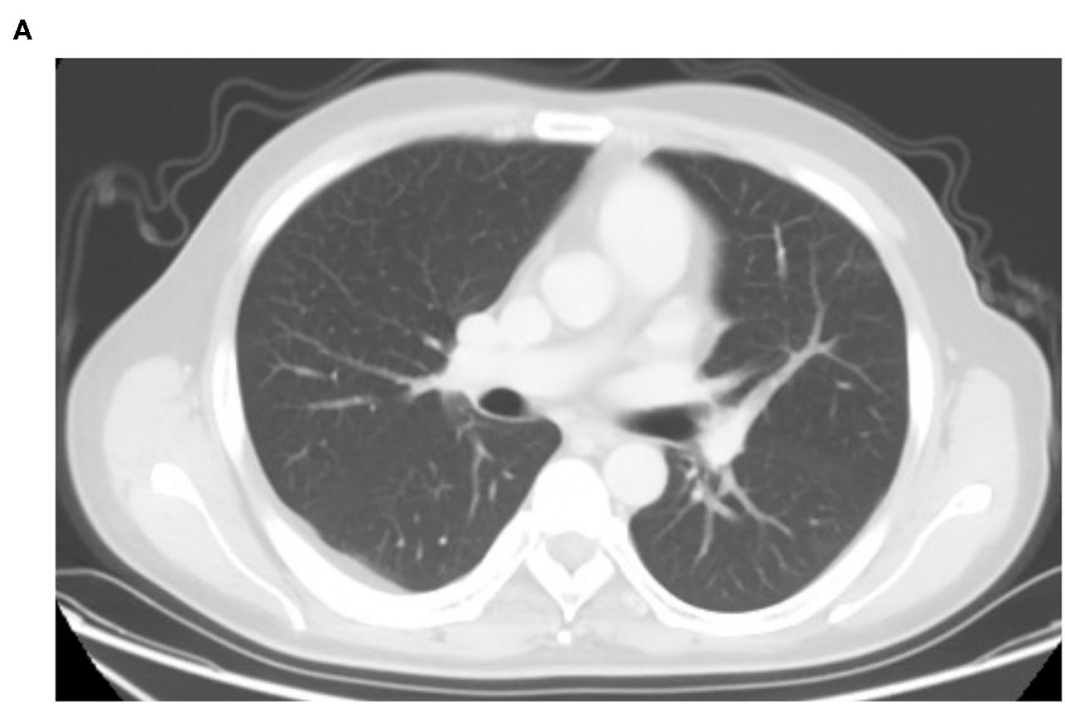

B

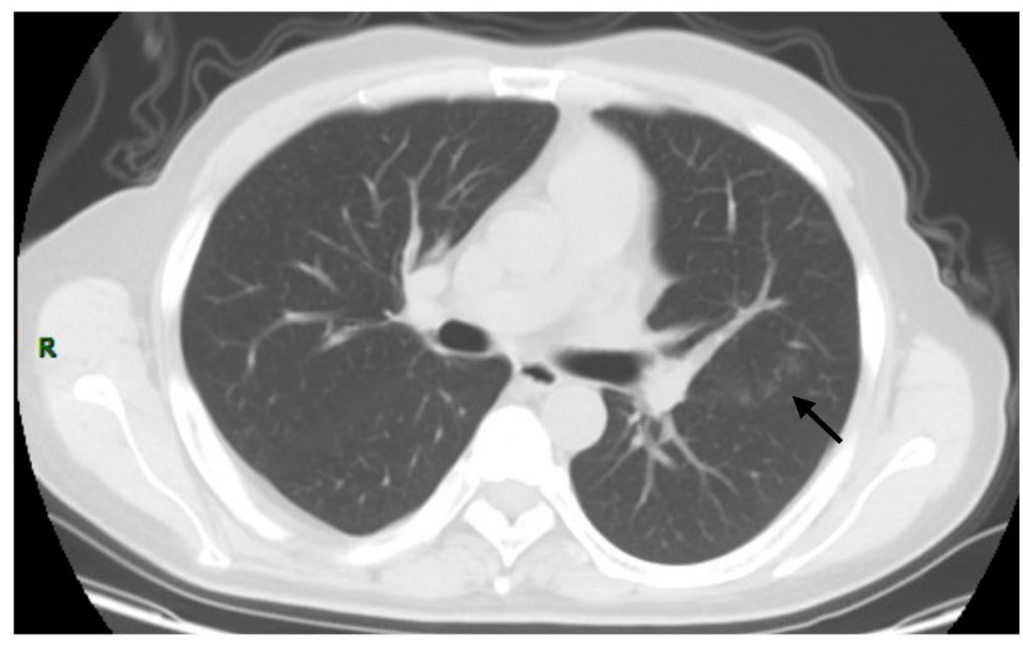

C

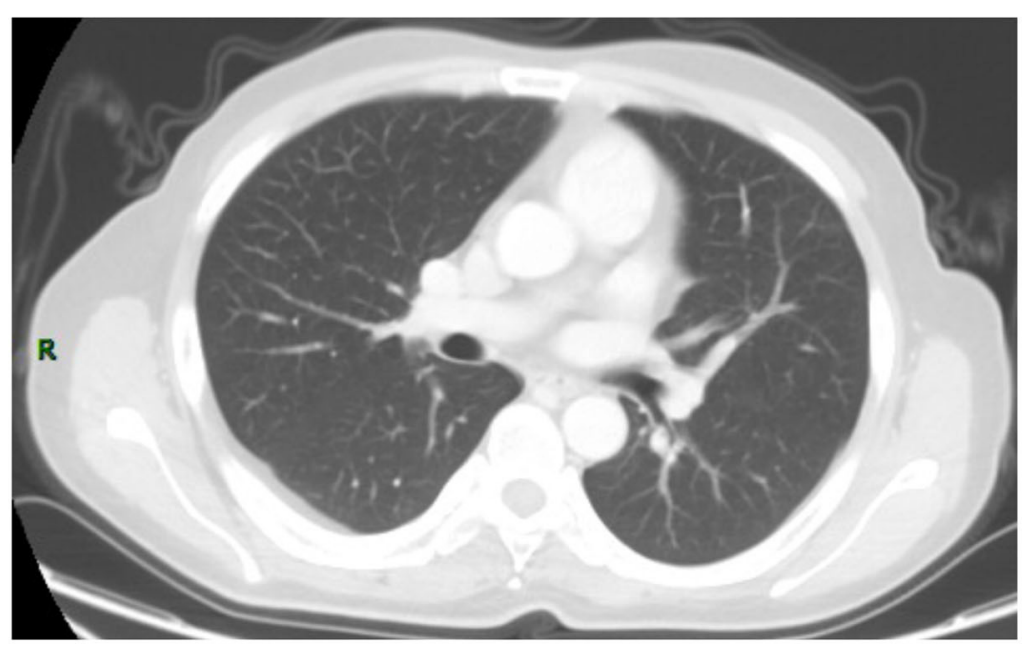

FIGURE 3 | Thirty-seven-year-old male with nasopharynx cancer. Patchy ground glass opacities affecting the left upper lobe had been newly seen (B) (arrow) when compared to the prior CT which performed at 2 months ago (A). The short-term CT scan (C) showed the lesion had been completely resolved. 


\section{A}

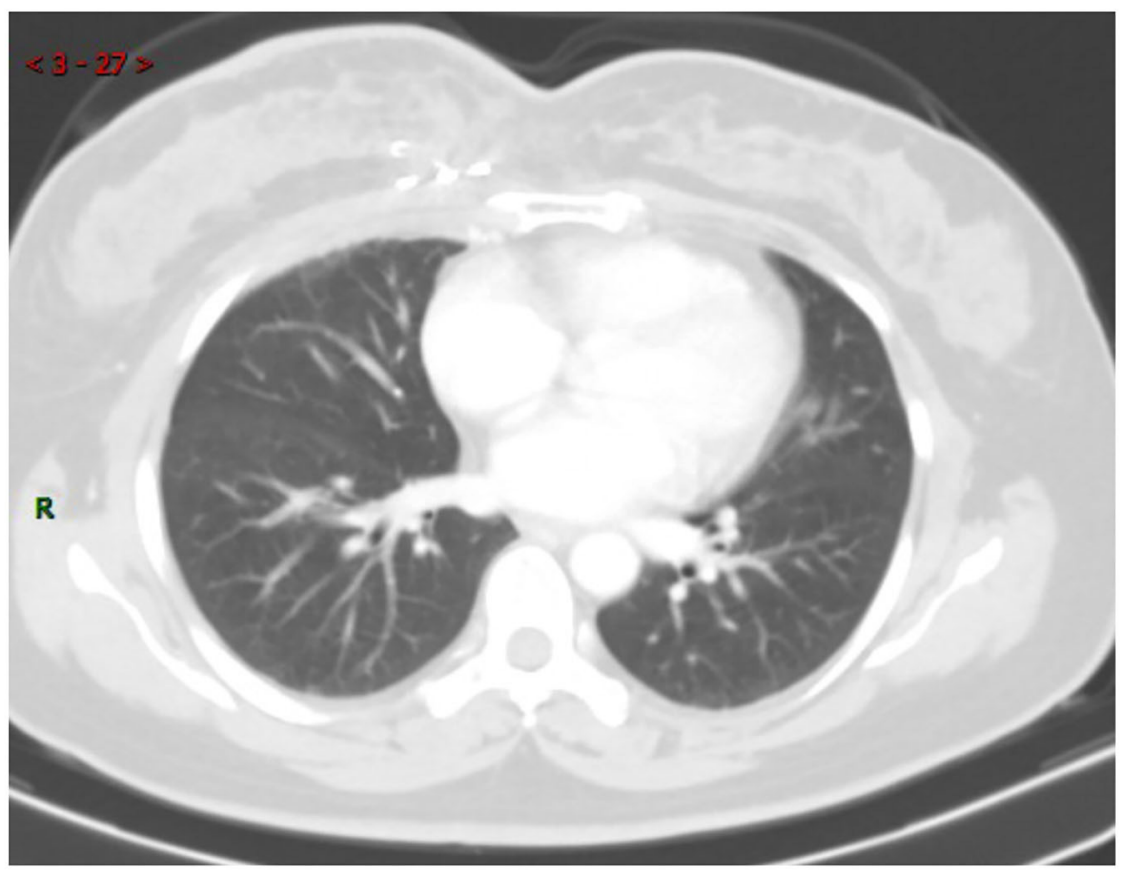

B

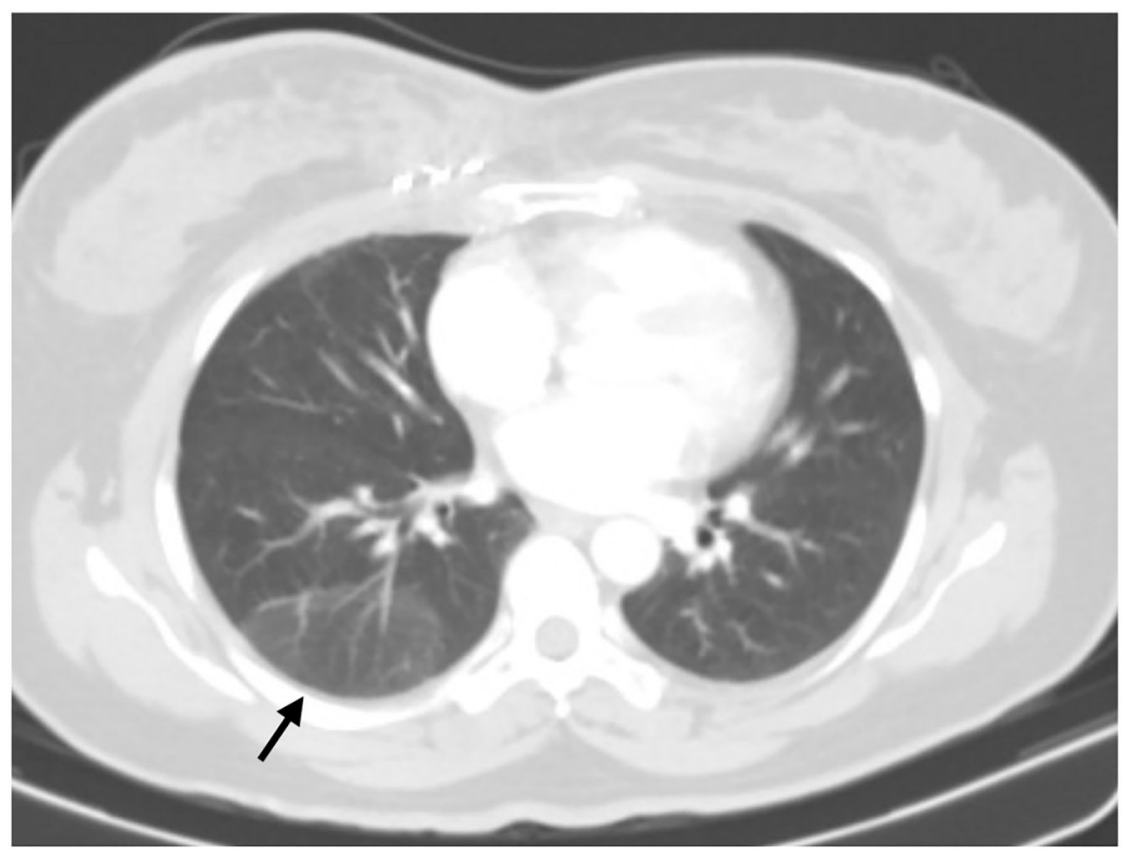

FIGURE 4 | Fourty-two-year-old female with breast cancer history. Screening CT scan showed a focal peripheral rounded and ill-defined GGOs in the right lower lobe [(B), arrow]. Comparison to the prior CT scan (A), it has been newly seen.

had increased in size (interval time: 8 days) compared with the previous CT scan.

Immediate RT-PCR was strongly recommended by the clinician for the patient, and the final test result was negative (Figure 7). Thirteen patients $(26.0 \%, 13 / 50)$ had no change (median: 19 days, range: 6-42 days). Among these, five patients $(18.5 \%, 5 / 27)$ showed cluster nodular lesion, three $(11.1 \%, 3 / 27)$ showed patchy shadow, most likely due to the inflammatory, three $(11.1 \%, 3 / 27)$ showed consolidation, most likely due to the inflammatory or atelectasis, one $(3.7 \%, 1 / 27)$ showed sub-pleural 


\section{A}

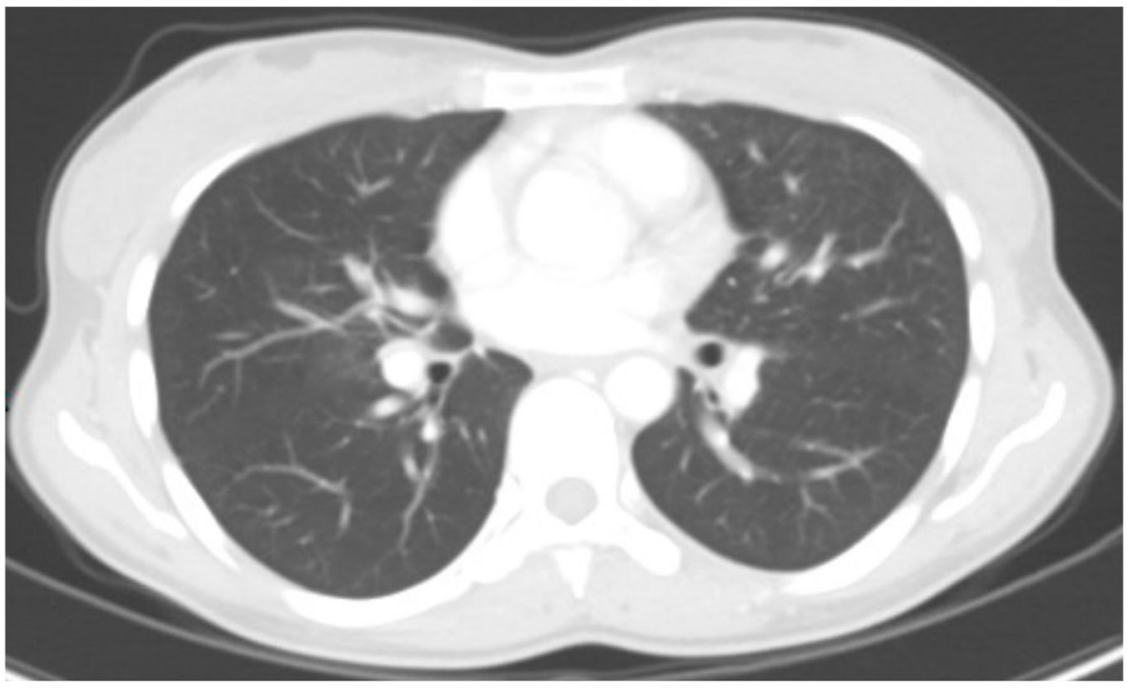

B

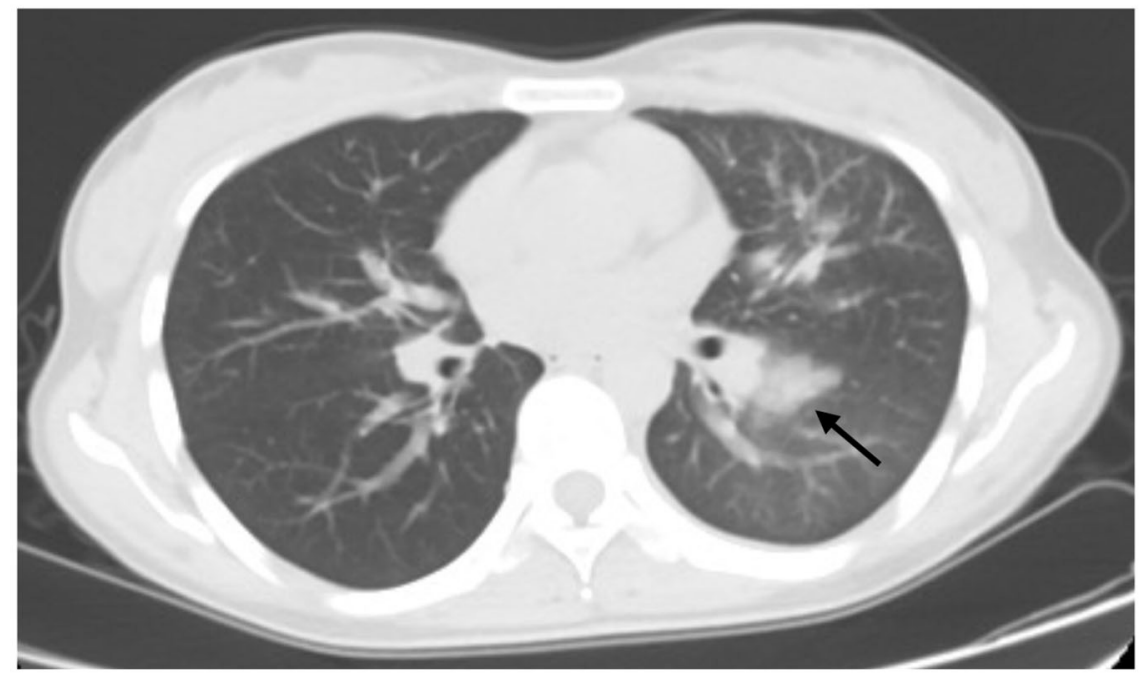

FIGURE 5 | Twenty-seven-year-old female with post-chemotherapy of lung cancer history. Comparison to the prior CT scan (A) which performed at 2 months ago, the focal left lower lobe subpleural consolidations was identified [(B), arrow].

interstitial inflammation change, and one $(3.7 \%, 1 / 27)$ manifested multiple GGO on bilateral lung parenchyma (Figure 8). Thirteen patients $(26.0 \%, 13 / 50)$ had resolved or partly resolved (median: 19 days, range: 6-53 days).

Among the 23 patients who had no further follow up CT scan, nine patients $(39.1 \%, 9 / 23)$ showed patchy shadow on sub-pleural due to atelectasis or hypostatic pneumonia, seven patients $(30.4 \%, 7 / 23)$ manifested consolidation due to chronic inflammation or atelectasis, four patients $(17.4 \%$, 4/23) manifested cluster of nodular lesion, most likely due to inflammatory, one $(4.3 \%, 1 / 23)$ manifested focal GGO, most likely due to Atypical adenomatous hyperplasia (AAH) of lung, one $(4.3 \%, 1 / 23)$ manifested segmental GGO, most likely due to inflammatory or $\mathrm{AAH}$, and another one $(4.3 \%, 1 / 23)$ manifested diffuse small airway lesions or respiratory bronchiolitis (All the COVID-19 screening CT finding were showed in Table 2).

\section{DISCUSSION}

At the time of writing, although COVID-19 is still spreading rapidly overseas, all COVID-19 patients in Wuhan, where the first case was reported in the city in December 2019, had been discharged, and hospitalized COVID-19 cases had dropped to zero on April 26, 2020. A new issue is that asymptomatic patients may add a new and worrying dimension to the spread of the pandemic. Besides Wuhan city, the medical institutions of all other Chinese cities have now switched back to their normal work status to serve the medical needs of the public. Because 

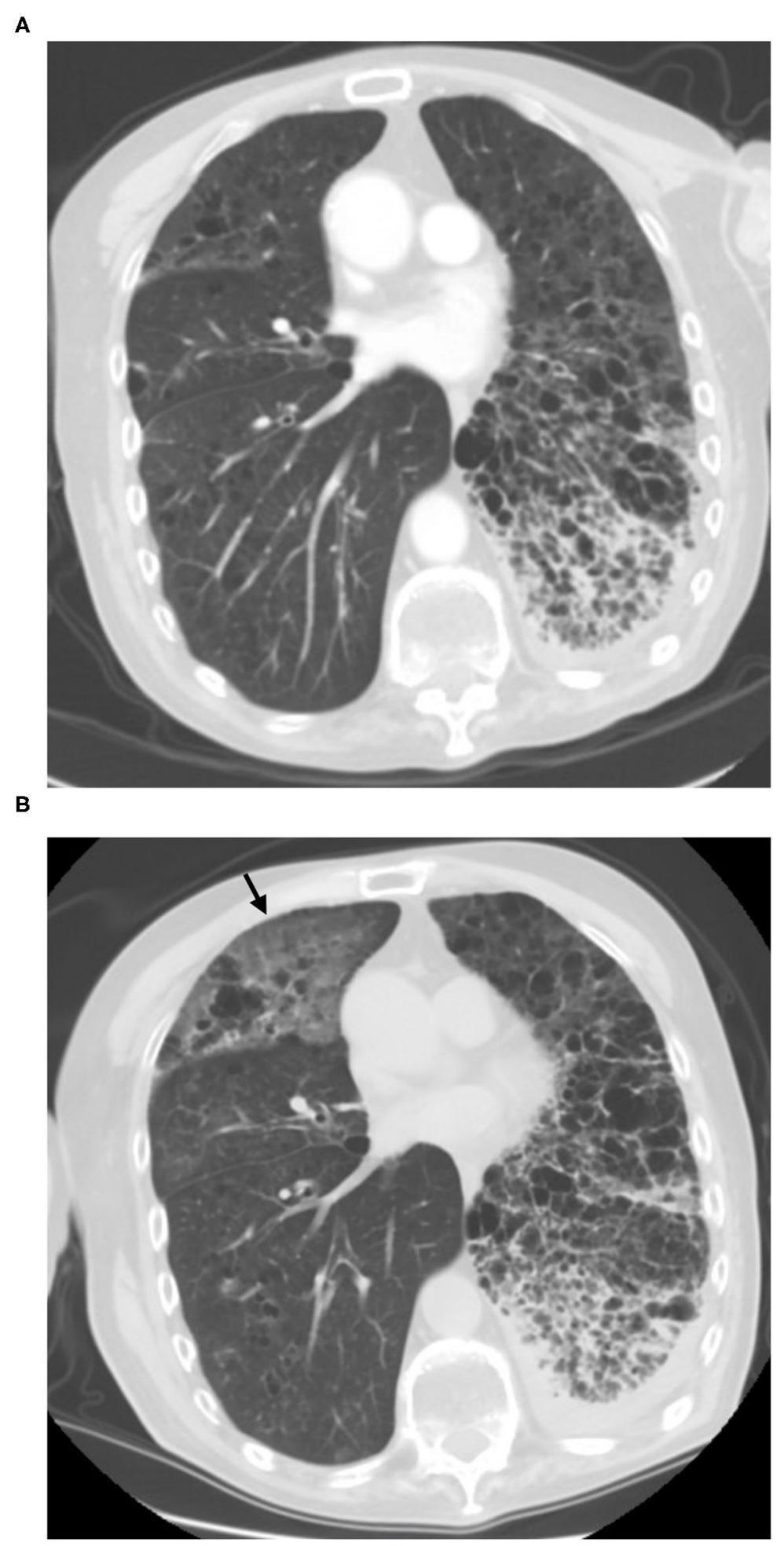

FIGURE 6 | Sixty-six-years-old male with post-target therapy of lung adenocarcinoma history. Comparison with the transverse CT scan (A), the screening CT (B) shows new bilateral, peripheral GGO associated with smooth interlobular and intralobular septal thickening (crazy-paving pattern) (arrow).

SARS-Cov-2 has proved to have the ability for efficient humanto human transmission, hospitals are not only gathering places for suspected COVID-19 patients but also remain high-risk areas for infection. From February 1, all patients, except emergencies, were denied access to our hospital. All the patients should make an appointment by telephone, internet, or hospital appointment 


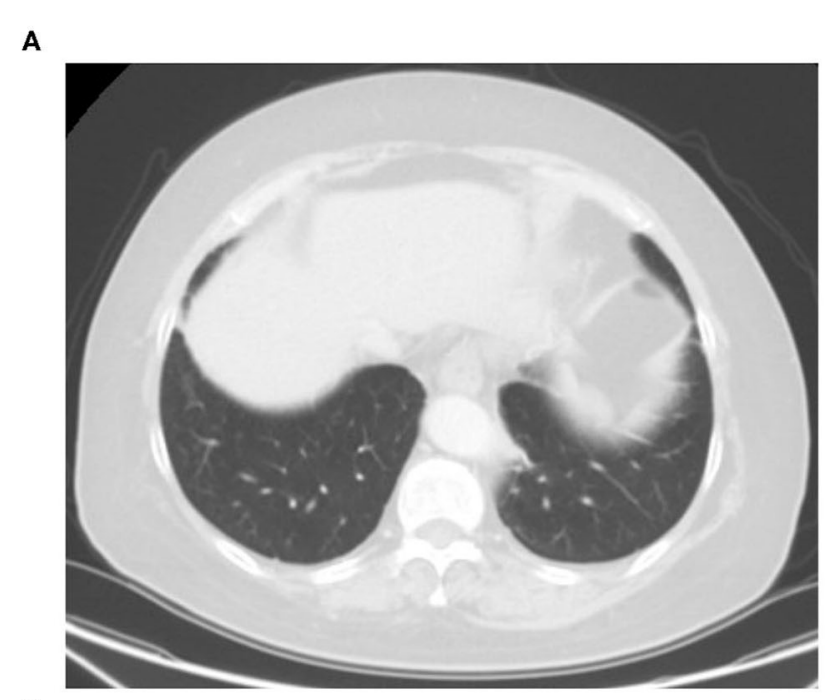

B

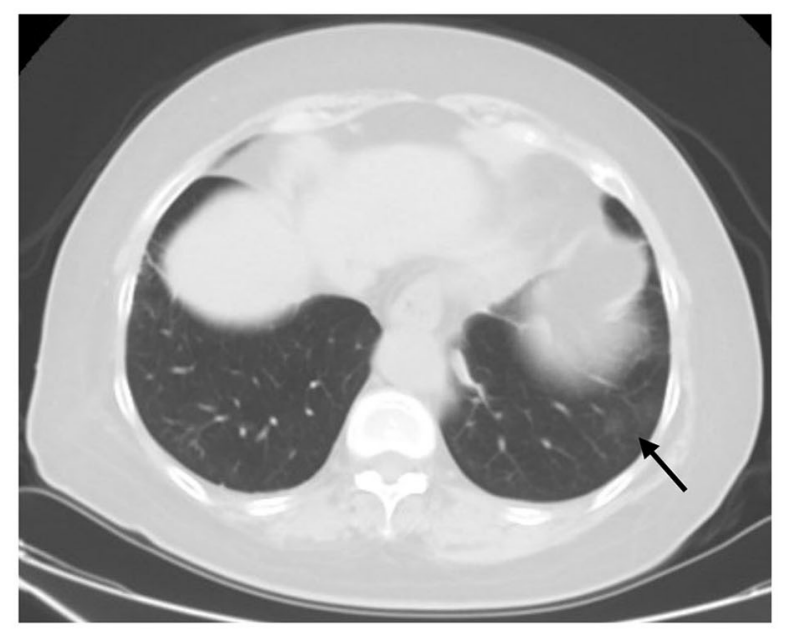

C

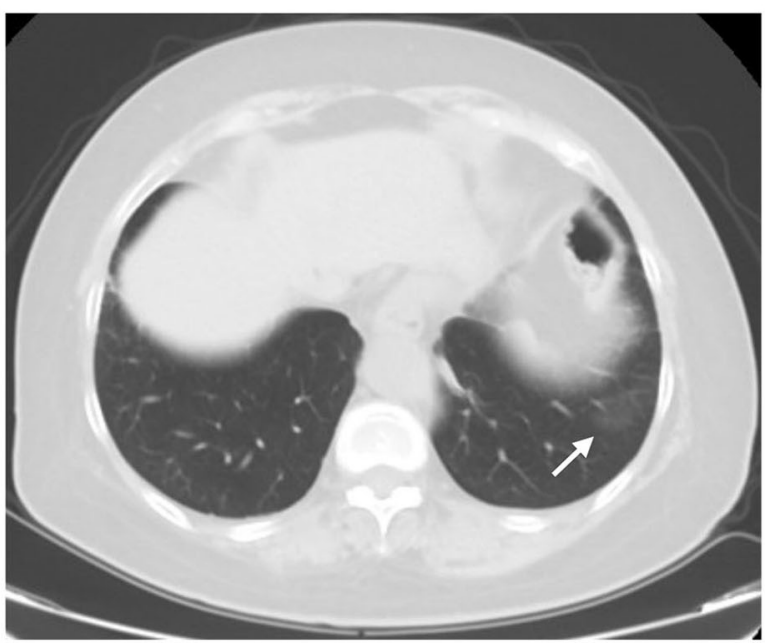

FIGURE 7 | Sixty-seven-year-old female with post-target therapy of lung cancer history. Screening CT scan (B) showed a pure GGO lesion can be newly seen in the left lower lobe (arrow) comparison to the prior CT scan which performed at 2 months ago (A). The short-term follow-up CT showed increases in the size of GGO in the lungs [(C), white arrow]. The RT-PCR results of the patient was negative. system. This minimized the risks to both patients and the healthcare team while reducing the utilization of unnecessary resources. All elective surgical and other anti-tumor treatments were postponed during the pandemic. In addition, postponing these services also minimized potential exposure of the COVID19 to unsuspecting healthcare providers and patients (15). The National Cancer Center of China, also known as the Cancer Hospital, is the largest cancer-specialist hospital and research institution in Asia. Nearly 1.3 million outpatients visit it, and nine thousand inpatients receive various anti-tumor-treatment. Therefore, to minimize the effect of the epidemic on cancer patients, providing recommendations of scientific and reasonable treatment and preventive measures for cancer patients in the global epidemic scenario is an urgent requirement. Given the occurrence of recurrent waves and outbreaks of COVID-19, it is of paramount importance to resume medical services in specialized hospitals in the long run while controlling the epidemic. Nowadays, patients were required to take the nucleic acid testing before receiving treatments or examinations in all specialized hospitals in China. However, this is not practical to be included as a clinical routine procedure. Accordingly, our experiences provided a feasible pathway for the specialized hospitals to deal with the potential threat of COVID-19 in the post-COVID 19 period. We recommend those who are suspect of COVID-19 from CT images to be referred to take the nucleic acid testing or other investigations. In this context, CT scans could serve as a timely alarm for further testing and intense follow-up so that we could provide the right care, to the right patients, at the right time.

When we decided to re-admit cancer patients, the most important consideration was identification of suspected cases of COVID-19 including patients and the accompanying persons at the earliest possible stage.

Although SARS-CoV-2 nucleic acid testing remains the golden standard for the diagnosis of COVID-19, due to qualification of the specimen sampling from nasopharyngeal swab and the detection kit, the positive rate of SARS-CoV-2 nucleic acid testing is only around $30-50 \%$ (16). High-resolution CT is the optimum choice to detect possible pulmonary opacities in COVID-19 suspicious patient. At present, low dose CT is not recommended for the screening of COVID-19 due to the low imaging quality and possibility of false negative GGO detection.

In order to detect suspected viral pneumonia even COVID19 on CT imaging, we have set up an effective mechanism of the supervisory radiologist reading system. The SR reads the initial CT images immediately after completion of the scan for each patient to determined suspected pneumonia CT characteristics. If the SR is unable to determine the pneumonia CT diagnosis, our procedure is to seek consultation from the PR. Fortunately, all the 50 cases we reported were false positive results which could be diagnosed by our thoracic-experience radiologists. To date, we have had no confirmed cases of COVID-19.

CT imaging characteristics are non-specific $(14,17)$. Although definitive diagnosis cannot be made on the basis of imaging features alone, especially in the COVID-19 low incidence area, in our cohort, the pneumonia lesions were predominantly peripheral and subpleural in $29(58.0 \%)$ patients, much less than other reports $(16,18-24)$. GGO and consolidation were 
A
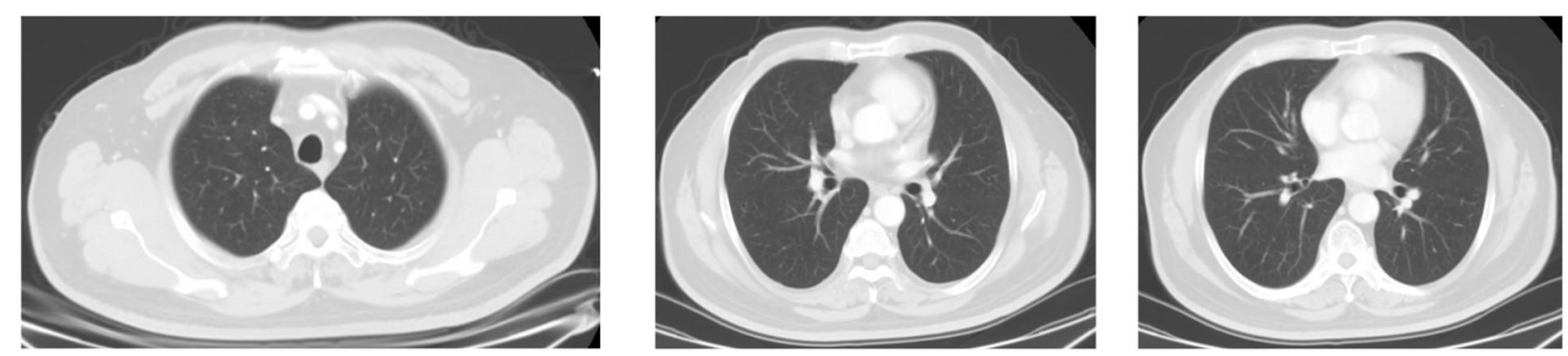

B
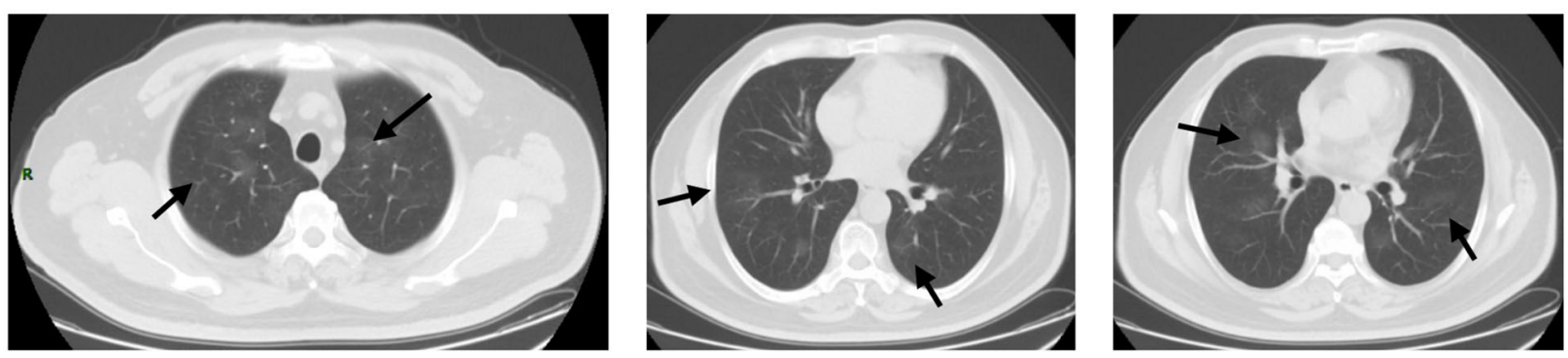

C
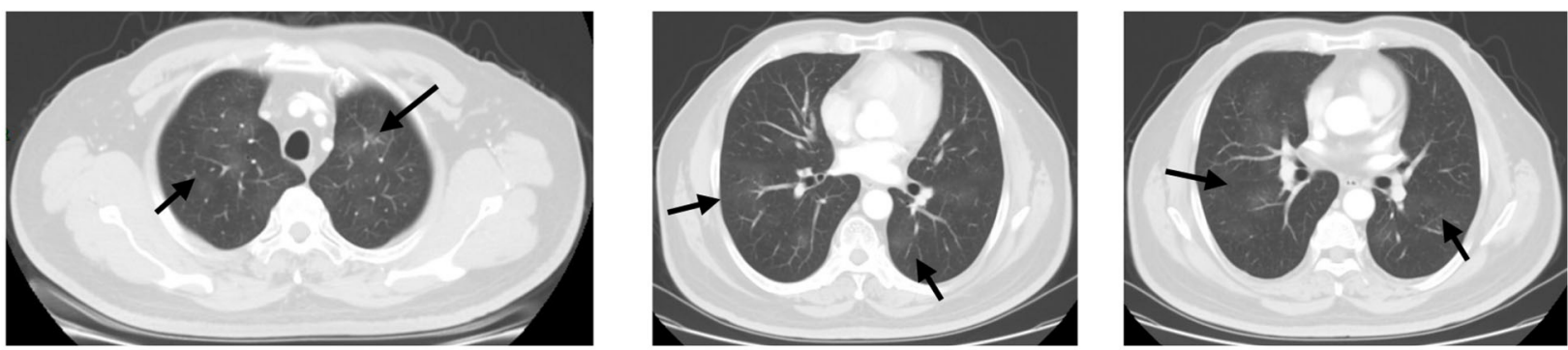

FIGURE 8 | Fifty-six-year-old male with post-operation of ascending colon carcinoma history. Comparison with the prior CT which performed 1 month ago (A), screening CT scans of the chest (B) show bilateral multiple GGOs without vascular enlargement (arrows). Most of lesions were identified in the centrally distributed. The short-term follow-up CT scan (C) showed unchanged (arrows). The RT-PCR results of the patient was negative.

manifested only in 14 cases, much lower than the typical COVID-19 CT finding on previously reports (16, 18-23). On the contrary, sub-pleura small patchy or strip-like lesions most likely due to fibrosis or hypostatic pneumonia and cluster of nodular lesions were two main signs of suspected cases on CT images (34,68.0\%). Therefore, we could distinguish these cases as the other type of pneumonia, like other viral pneumonia, bacterial pneumonia, mycoplasma pneumonia, and many non-infectious diseases preliminarily from the COVID19 cases. Machine-learning based methods are emerging rapidly in recent years focusing on COVID detection or disease diagnosis $(24,25)$. These methods may be helpful to differentiate COVID-19 from other pulmonary infection and/or noninfectious diseases $(26,27)$. However, our experience would provide a practical tool for health providers where machine learning is not accessible, especially in hospitals with limited medical resources.
TABLE 2 | Morphological feature of screening CT and the results of follow-up CT scan.

\begin{tabular}{|c|c|c|c|c|}
\hline & \multicolumn{3}{|c|}{ Patients had further CT scan $(n=27)$} & \multirow{2}{*}{$\begin{array}{l}\text { Patients had no } \\
\text { further CT scan } \\
\qquad(n=23)\end{array}$} \\
\hline & $\begin{array}{l}\text { Unchanged } \\
\qquad(n=13)\end{array}$ & $\begin{array}{l}\text { Resolve or } \\
\text { partly resolve } \\
(n=13)\end{array}$ & $\begin{array}{l}\text { Increase } \\
\text { in size } \\
(n=1)\end{array}$ & \\
\hline $\begin{array}{l}\text { Cluster nodular } \\
\text { lesion }\end{array}$ & 5 & 7 & 1 & 4 \\
\hline GGO & 1 & 0 & 0 & 2 \\
\hline $\begin{array}{l}\text { Interstitial } \\
\text { inflammation }\end{array}$ & 1 & 0 & 0 & 0 \\
\hline Consolidation & 3 & 1 & 0 & 7 \\
\hline $\begin{array}{l}\text { Sub-plerual } \\
\text { patchy } \\
\text { shadow }\end{array}$ & 3 & 5 & 0 & 9 \\
\hline $\begin{array}{l}\text { Respiratory } \\
\text { bronchiolitis }\end{array}$ & 0 & 0 & 0 & 1 \\
\hline
\end{tabular}


Some patients with COVID-19 who do not receive effective therapy will demonstrate deterioration and changes will be relatively rapid. The lesions on CT will become more diffuse distributed in a truly short term $(28,29)$. Therefore, serial CT imaging of patients could help to diagnosis, or continuously monitor disease changes. In our cohort, 13 patients $(26.0 \%)$ had no change, while 13 patients $(26.0 \%)$ had resolved or partly resolved. These cases were subsequently excluded by the diagnosis of COVID-19 because of persistent existence pulmonary lesions or resolved in a short-period. Only one patient had increased in size (interval time: 8 days) compared with the further CT scan and the final RT-PCR test result was negative.

\section{LIMITATIONS}

The study has several limitations. First, this was a single-center study, and a multicenter study and/or including more cases, especially more confirmed COVID-19 cases, are needed.

Second, we did not take throat swab samples and the blood routine examination of WBC, Neutrophil, and Lymphocyte are more suitable for finding cancer patients with asymptomatic suspected COVID-19 infection. Third, 23 (46.0\%) patients had no further CT examination to evaluate the change of the CT feature. Therefore, long-term radiological follow-up is needed to confirm our findings.

\section{CONCLUSION}

In conclusion, CT may be useful as a screening tool for COVID19 based on imaging features. Confirmed diagnosis should,

\section{REFERENCES}

1. World Health Organization. WHO Director-General's opening remarks at the media briefing on COVID-19-11 March. (2020). Available online at: https:// www.who.int/dg/speeches/detail/who-director-general-s-opening-remarksat-the-media-briefing-on-covid-19-11-march-2020

2. Ren L, Wang Y, Wu Z, Xiang Z, Guo L, Xu T, et al. Identification of a novel coronavirus causing severe pneumonia in human: a descriptive study. Chin Med J. (2020) 133:1015-24. doi: 10.1097/CM9.0000000000000722

3. Chan JF, Yuan S, Kok KH, To KK, Chu H, Yang J, et al. A familial cluster of pneumonia associated with the 2019 novel coronavirus indicating personto-person transmission: a study of a family cluster. Lancet. (2020) 395:51423. doi: 10.1016/S0140-6736(20)30154-9

4. Wang C, Horby PW, Hayden FG, Gao GF, A. novel coronavirus outbreak of global health concern. Lancet. (2020) 395:4703. doi: 10.1016/S0140-6736(20)30185-9

5. Huang C, Wang Y, Li X, Ren L, Zhao J, Hu Y, et al. Clinical features of patients infected with 2019 novel coronavirus in Wuhan, China. Lancet. (2020) 395:497-506. doi: 10.1016/S0140-6736(20)30183-5

6. World Health Organization. Global Surveillance for COVID-19 Caused by Human Infection With COVID-19 Virus: Interim Guidance. Geneva: World Health Organization (2020). Available online at: http://apps.who.int/iris/rest/ bitstreams/1272502/retrieve

7. China National Health Commission website. Notice on issuing a new coronavirus infected pneumonia diagnosis and treatment plan. Available online at: (trial version 5) Bgs.satcm.gov.cn/zhengcewenjian/2020-02-06/12847.html (accessed February 18, 2020). however, ultimately be based on the etiology. The cancer patients in low-incidence areas should continue treatment after careful screening before admission.

\section{DATA AVAILABILITY STATEMENT}

The raw data supporting the conclusions of this article will be made available by the authors, without undue reservation.

\section{AUTHOR CONTRIBUTIONS}

WT: conceptualization, data curation, formal analysis, investigation, methodology, writing-original draft, and writingreview and editing. NW and J-WW: conceptualization, formal analysis, investigation, methodology, supervision, funding acquisition, project administration, writing-original draft, and writing-review and editing. XZ, S-JZ, LL, and $\mathrm{YH}$ : formal analysis, investigation, and writing-review and editing. All authors contributed to the article and approved the submitted version.

\section{FUNDING}

This study was supported by the National Key Research and Development Program of China (No. 2017YFC1308700), National Natural Sciences Foundation of China (No. 81971616), Beijing Nova Program (Z202200006820070), and Chinese Academy of Medical Sciences Initiative for Innovative Medicine (No. 2017-I2M-1-005).

8. Ai T, Yang Z, Hou H, Zhan C, Chen C, Lv W, et al. Correlation of chest CT and RT-PCR testing in coronavirus disease 2019 (COVID-19) in China: a report of 1014 cases. Radiology. (2020) 26:200642. doi: 10.1148/radiol.2020200642

9. Bai HX, Hsieh B, Xiong Z, Halsey K, Choi JW, Tran TML, et al. Performance of radiologists in differentiating COVID-19 from viral pneumonia on chest CT. Radiology. (2020) 10:200823. doi: 10.1148/radiol.2020200823

10. National Health Commission of the People's Republic of China website. Diagnosis and treatment of novel coronavirus infection. Available online at: http://www.nhc.gov.cn/yzygj/s7653p/202002/ 8334a8326dd94d329df351d7da8aefc2.shtml (accessed February 19, 2020).

11. Guan W-J, Ni Z-Y, Hu Y, Liang W-H, Ou C-Q, He J-X, et al. Clinical characteristics of coronavirus disease 2019 in China. N Engl J Med. (2020) 382:1708-20. doi: 10.1056/NEJMoa2002032

12. Liang W, Guan W, Chen R, Wang W, Li J, Xu K, et al. Cancer patients in SARS-CoV-2 infection: a nationwide analysis in China. Lancet Oncol. (2020) 21:335-7. doi: 10.1016/S1470-2045(20)30096-6

13. Ajlan AM, Ahyad RA, Jamjoom LG, Alharthy A, Madani TA. Middle East respiratory syndrome coronavirus. (MERS-CoV) infection: chest CT findings. AJR Am J Roentgenol. (2014) 203:782-7. doi: 10.2214/AJR.14.13021

14. Joint GI Society Message: COVID-19 clinical insights for our community of gastroenterologists and gastroenterology care providers. Available online at: https://www.asge.org/home/joint-gi-society-message-covid-19 (accessed March 23, 2020).

15. Xie X, Zhong Z, Zhao W, Zheng C, Wang F, Liu J. Chest CT for typcal 2019nCoV pneumonia: relationship to negative RT-PCR testing. Radiology. (2020) 296:200343. doi: 10.1148/radiol.2020200343

16. Das KM, Lee EY, Enani MA, AIJawder SE, Singh R, Bashir S, et al. CT correlation with outcomes in 15 patients with acute Middle East 
respiratory syndrome coronavirus. AJR Am J Roentgenol. (2015) 204:73642. doi: 10.2214/AJR.14.13671

17. Shi H, Han X, Fan Y, et al. Radiologic features of patients with 2019-nCoV infection. J Clin Radiol. (2020) 39:8-11. doi: 10.13437/j.cnki.jcr.20200206.002

18. Huang L, Han R, Yu P-X, Wang S-K, Xia L-M. A correlation study of CT and clinical features of different clinical types of COVID-19. Chin J Radiolo. (2020) 54:300-4.

19. Liu P, Tan X. Novel coronavirus (2019-nCoV) pneumonia. Radiology. (2020) 295:19. doi: 10.1148/radiol.2020200257

20. Kanne JP. Chest CT findings in 2019 novel coronavirus. (2019-nCoV) infections from Wuhan. China: key points for the radiologist. Radiology. (2020) 295:16-7. doi: 10.1148/radiol.2020200241

21. Shi H, Han X, Zheng C. Evolution of CT manifestations in a patient recovered from 2019 novel coronavirus. (2019-n-CoV) pneumonia in Wuhan. China Radiology. (2020) 295:20. doi: 10.1148/radiol.2020 200269

22. Qian L, Yu J, Shi H. Severe acute respiratory disease in a Huanan seafood market worker: images of an early casualty. Radiol Cardiothorac Imaging. (2020) 2:e200025. doi: 10.1148/ryct.2020200033

23. Huang $P$, Liu $T$, Huang L, Liu $H$, Lei $M$, Xu $W$, et al. Use of chest CT in combination with negative RT-PCR assay for the 2019 novel coronavirus but high clinical suspicion. Radiology. (2020) 295:223. doi: 10.1148/radiol.2020200330

24. Roberts M, Driggs D, Thorpe M, Gilbey J, Schnlieb CB. Common pitfalls and recommendations for using machine learning to detect and prognosticate for COVID-19 using chest radiographs and CT scans. Nature Machine Intelligence. (2021) 3:199-217. doi: 10.1038/s42256-021-00307-0

25. Driggs D, Selby I, Roberts M, Gkrania-Klotsas E, Schnlieb C. Machine learning for COVID-19 diagnosis and prognostication: lessons for amplifying the signal while reducing the noise. Radiology. (2021) 3: e210011. doi: 10.1148/ryai.2021210011

26. Hu, S, Gao Y, Niu Z, Jiang Y, Yang G. Weakly supervised deep learning for covid-19 infection detection and classification from CT images. IEEE Access. (2020) 8:118869-83. doi: 10.1109/ACCESS.2020. 3005510

27. Ma H, Ye Q, Ding W, Jiang Y, Yang G. Can clinical symptoms and laboratory results predict CT abnormality? initial findings using novel machine learning techniques in children with COVID19 infections. Front Med. (2021) 8:855. doi: 10.3389/fmed.2021. 699984

28. Jiang N-C, Zheng C-S, Fan Y-Q, Han X-Y, Chen Y, Cheng Q-G, et al. CT appearances and short-term, changes of COVID-19 in subclinical period. Chin J Radiolo. (2020) 54:305-9.

29. Shohei N, Yusuke K, Yuya A, Sho F, Masaru T, Kazuaki F, et al. Characteristics and outcomes of c coronavirus disease 2019(COVID-19) patients with cancer: a single-center retrospective observational study in Tokyo, Japan. Int J Clin Oncol. (2021) 23:485-93 doi: 10.1007/s10147-020-01837-0

Conflict of Interest: The authors declare that the research was conducted in the absence of any commercial or financial relationships that could be construed as a potential conflict of interest.

Publisher's Note: All claims expressed in this article are solely those of the authors and do not necessarily represent those of their affiliated organizations, or those of the publisher, the editors and the reviewers. Any product that may be evaluated in this article, or claim that may be made by its manufacturer, is not guaranteed or endorsed by the publisher.

Copyright (C) 2021 Tang, Wang, Wang, Huang, Liu, Zhao, Zhao and Wu. This is an open-access article distributed under the terms of the Creative Commons Attribution License (CC BY). The use, distribution or reproduction in other forums is permitted, provided the original author(s) and the copyright owner(s) are credited and that the original publication in this journal is cited, in accordance with accepted academic practice. No use, distribution or reproduction is permitted which does not comply with these terms. 